\title{
HIV-based lentiviral vectors for anti-HIV gene therapy
}

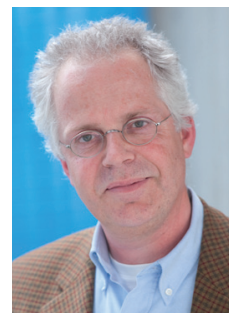

"There is reason to believe that LVs are safer than retroviral vectors because LVs do not favor integration near the start site of genes, thus restricting their potential to upregulate cellular gene expression."

Ben Berkhout

Laboratory of Experimental Virology, Department of Medical Microbiology, Center for Infection \& Immunity Amsterdam (CINIMA), Academic Medical Center, University of Amsterdam, K3-110, Meibergdreef 15, 1105 AZ Amsterdam, The Netherlands = Tel. : +31 205664822 = Fax: +31 206916531 m b.berkhout@amc.uva.nl

HIV-1 research has been very successful in terms of development of antiretroviral drugs, but the design of an effective vaccine that protects against infection is still in very early days. Antiretroviral therapy remains problematic in some cases, for example, owing to limited drug accessibility and/or the cost involved, poor drug adherence and, consequently, the development of viral resistance, or long-term toxicity that necessitates a switch in the drug regimen. Therefore, there is a need for the development of novel therapeutic approaches that should provide a more durable therapeutic effect, and gene therapy is a promising candidate. In this article, I will focus on the advantages and disadvantages of using a HIV-based lentiviral vector (LV) for therapeutic intervention in HIV-1 patients.

The LV system has many advantages that make it particularly attractive for the design of an anti-HIV therapy. First, LVs can transduce nondividing cells; a property that makes them more appropriate for many clinical applications than the early retroviral vectors. In particular, LVs that are pseudotyped with the vesicular stomatitis virus glycoprotein can efficiently transduce $\mathrm{CD} 34^{+}$hematopoietic precursor cells or $\mathrm{CD}^{+}$mature T cells, which form the major target cell populations for anti-HIV gene therapy. In other words, gene delivery is no longer the bottle neck; although other problems remain, such as how to reach a sufficient number of cells in an ex vivo gene therapy setting. Second, LVs will stably deposit their antiviral cargo in the host cell genome, which is very attractive if one wants to impose a lifelong therapeutic effect. This integration event occurs at a random position on one of the human chromosomes, which obviously raises safety concerns. The induction of leukemia by integration of retroviral vectors near a cellular proto-oncogene has been well documented. There is reason to believe that LVs are safer than retroviral vectors because LVs do not favor integration near the start site of genes, thus restricting their potential to upregulate cellular gene expression. In addition, dangerous transcriptional enhancing elements have been removed in the modern (self-inactivating) LV design. Although it is still early days and only a few clinical trials have been performed, no adverse effects of LV integration have been reported so far, and particularly promising is the durable therapeutic effect observed in the brain of adrenoleukodystrophy patients, when the $\mathrm{CD} 34^{+}$cells were treated ex vivo with a LV encoding a therapeutic gene that is subsequently expressed by microglial cells in the brain [1]. In particular, for the treatment of HIV-infected individuals, where viral integration events occur in very large numbers every day, this safety aspect could possibly be relaxed. In order to reduce the risk of insertional oncogenesis for other LV applications, there is much research activity to generate nonintegrating LVs, but their efficiency is significantly reduced compared with integrating LVs.

Many different types of antiviral cargo can be shipped by the LV vector system, including genes that encode inhibitory proteins such as transdominant negative mutant HIV-1 proteins or diverse RNA transcripts with antiviral activity. The latter class includes decoy elements, such as the HIV-1 TAR hairpin motif, which absorb important viral and/or cellular proteins, antisense transcripts that directly target the HIV-1 RNA genome, or ribozymes that cleave HIV-1 RNA or a cellular mRNA that encodes a critical cofactor such as the CCR5 receptor. Perhaps the most promising addition to the antiviral arsenal are inhibitors based on the cellular RNAi mechanism. Transient therapy with synthetic siRNA is currently in clinical trials for treatment of acute respiratory viral infections [2], but a durable

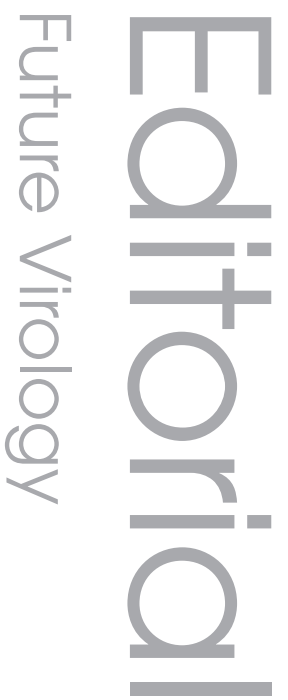

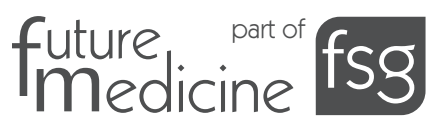


anti-HIV therapy would probably require a more long-lasting gene therapy approach [3]. RNAi gene cassettes that encode shRNA or miRNAlike transcripts can be incorporated in the LV. The potency and strict sequence specificity of antiviral RNAi approaches was demonstrated by the selection of resistant HIV-1 variants that acquire a single mutation in the 19-nucleotide target sequence $[4,5]$. To achieve lasting virus suppression, combinatorial RNAi approaches have subsequently been developed with multiple shRNA cassettes, polyvalent miRNA transcripts or extended shRNA [6-8].

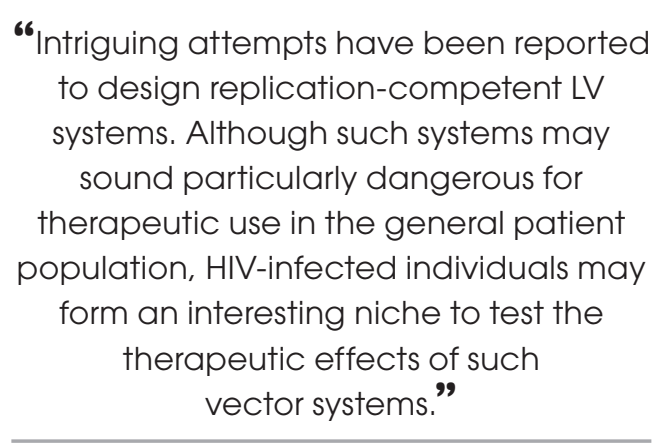

The titer of therapeutic LV vectors may decrease as a result of the insert size, certain RNA sequences and/or structures. RNAi cassettes may cause specific problems. Any RNAi inhibitor will also be expressed in the LV producer cell, where it can cause self-targeting and degradation of the vector RNA genome. We demonstrated that this disastrous effect does not readily occur for shRNA cassettes because the stable hairpin structure will protect the target sequence against RNAi attack [9]. miRNA cassettes, when inserted in the sense orientation, may also destabilize the vector RNA genome when it is recognized and cleaved by the Drosha enzyme $[10,11]$. A particular problem arises when sequence elements, such as polymerase III promoters to drive the expression of multiple shRNAs, are repeated in the LV context. Such repeats cause massive recombination-mediated deletions during reverse transcription, such that therapeutic units are lost upon transduction, frustrating the combinatorial approach [12].

Antiviral reagents that target HIV-1 genome sequences may obviously conflict with the use of the LV system that may contain the identical HIV-1 sequences. The vector genome itself does contain relatively few HIV-1 sequences, but the complete coding information for the HIV-1 Gag-Pol and Rev proteins is present on other plasmids of the LV system. To avoid vectortargeting of the LV system, one could choose target sequences that are not represented in the LV plasmids, for example Env-encoding sequences. Alternatively, one could use a 'codonoptimized' version of the Gag-Pol plasmid that has been around for some time.

The first LV clinical trial in HIV-infected patients was initiated recently by the team of John Rossi at the City of Hope (CA, USA). The antiviral cocktail used in this trial consists of a TAR RNA decoy, CCR5 ribozyme and antiHIV shRNA. It is predicted that the modified cells will have a major survival benefit over nontransduced cells, which will readily be infected by HIV-1 and subsequently removed by the immune system. An unexpected proof of concept of this therapeutic strategy was presented by the story of the 'Berlin patient' [13]. An HIVpositive man was diagnosed in 2006 with acute leukemia. In an attempt to treat the leukemia, the doctor arranged for him to receive a stem cell transplant from a donor who did not express a functional CCR5 protein. Ever since that transplant, which remains a high-risk procedure, the Berlin patient has had an undetectable viral load despite not being on antiretroviral therapy. The man has generously allowed scientists to take almost every possible biopsy and tests, including the most ultrasensitive HIV test available, indicates that HIV is not detectable anywhere in his body. It would seem much too early to state that the virus has been eradicated from this patient, but the virus apparently finds no cells to fuel its replication, and one could call this a functional cure. It is now almost 3 years since this operation and HIV still seems not to have reemerged. This story inspires new hope that some sort of gene therapy, such as inducing CCR5 silencing by means of RNAi $[14,15]$, may be the key to an HIV-1 cure.

Intriguing attempts have been reported to design replication-competent LV systems. Although such systems may sound particularly dangerous for therapeutic use in the general patient population, HIV-infected individuals may form an interesting niche to test the therapeutic effects of such vector systems. In fact, one replicating vector is already being evaluated in a clinical trial, the LV encoding an extended Env antisense segment that was developed by the VIRxSYS company (MD, USA) [16]. To control the replication potential of such vectors, doxycycline-dependent lentiviral genomes have been designed [17]. Antiviral RNAi cassettes that target wild-type HIV-1 sequences that were removed from the vector were incorporated in this inducible vector, thus avoiding self-targeting [18]. 
Unlike nonreplicating vector systems, replicating LVs encode all essential viral proteins and, thus, may open up possibilities for combining antiviral therapy with vaccination strategies.

\section{Financial \& competing interests disclosure}

The author has a patent pending for 'Means and methods for durable inhibition of pathogens' (EP09153919.7).

Work in the author's laboratory is supported by
ZonMw Translational Gene Therapeutic Grant. The author has no other relevant affliations or financial involvement with any organization or entity with a financial interest in or financial conflict with the subject matter or materials discussed in the manuscript apart from those disclosed.

No writing assistance was utilized in the production of this manuscript.

\section{Bibliography}

1. Cartier N, Hacein-Bey-Abina S, Bartholomae CC et al.: Hematopoietic stem cell gene therapy with a lentiviral vector in X-linked adrenoleukodystrophy. Science 326(5954), 818-823 (2009).

2. DeVincenzo J, Cehelsky JE, Alvarez R et al.: Evaluation of the safety, tolerability and pharmacokinetics of ALN-RSV01, a novel RNAi antiviral therapeutic directed against respiratory syncytial virus (RSV). Antiviral Res. 77(3), 225-231 (2008).

3. Berkhout B: Toward a durable anti-HIV gene therapy based on RNA interference. Ann. NY Acad. Sci. 1175, 3-14 (2009).

4. Boden D, Pusch O, Lee F, Tucker L, Ramratnam B: Human immunodeficiency virus type 1 escape from RNA interference. J. Virol. 77(21), 11531-11535 (2003).

5. Das AT, Brummelkamp TR, Westerhout EM et al: Human immunodeficiency virus type 1 escapes from RNA interference-mediated inhibition. J. Virol. 78(5), 2601-2605 (2004).

6. Liu YP, Berkhout B: Lentiviral delivery of RNAi effectors against HIV-1. Curr. Top. Med. Chem. 9(12), 1130-1143 (2009).

7. Barichievy S, Saayman S, Arbuthnot P, Weinberg MS: RNA interference-based gene expression strategies aimed at sustained therapeutic inhibition of HIV.

Curr. Top. Med. Chem. 9(12), 1065-1078 (2009).

8. Zhang J, Rossi JJ: Strategies in designing multigene expression units to downregulate HIV-1. Methods Mol. Biol. 623, 123-136 (2010).

9. Westerhout EM, Berkhout B: A systematic analysis of the effect of target RNA structure on RNA interference. Nucleic Acids Res. 35(13), 4322-4330 (2007).

10. Liu YP, Vink M, Westerink JT et al.: Titers of lentiviral vectors encoding shRNAs and miRNAs are reduced by different mechanisms that require distinct repair strategies. RNA 16(7), 1328-1339 (2010).

11. Liu YP, von Eije KJ, Schopman NC et al.: Combinatorial RNAi against HIV-1 using extended short hairpin RNAs. Mol. Ther. 17(10), 1712-1723 (2009).

12. Ter Brake O, Konstantinova P, Ceylan M, Berkhout B: Silencing of HIV-1 with RNA interference: a multiple shRNA approach. Mol. Ther. 14(6), 883-892 (2006).

13. Hutter G, Nowak D, Mossner M et al:: Long-term control of HIV by CCR5 $\Delta 32 / \Delta 32$ stem-cell transplantation. $N$. Engl. J. Med. 360 (7), 692-698 (2009).
14. Martinez MA, Gutierrez A, Armand-Ugon M et al.: Suppression of chemokine receptor expression by RNA interference allows for inhibition of HIV-1 replication. AIDS 16(18), 2385-2390 (2002).

15. Shimizu S, Hong P, Arumugam B et al.: A highly efficient short hairpin RNA potently down-regulates CCR5 expression in systemic lymphoid organs in the hu-BLT mouse model. Blood 115(8), 1534-1544 (2010).

16. Lu X, Yu Q, Binder GK et al.: Antisense-mediated inhibition of human immunodeficiency virus (HIV) replication by use of an HIV type 1-based vector results in severely attenuated mutants incapable of developing resistance. J. Virol. 78(13), 7079-7088 (2004).

17. Marzio G, Verhoef K, Vink M, Berkhout B: In vitro evolution of a highly replicating, doxycycline-dependent HIV for applications in vaccine studies. Proc. Natl Acad. Sci. USA 98(11), 6342-6347 (2001).

18. Westerhout EM, Vink M, Haasnoot PC, Das AT, Berkhout B: A conditionally replicating HIV-based vector that stably expresses an antiviral shRNA against HIV-1 replication. Mol. Ther. 14(2), 268-275 (2006). 